\title{
e-Learning System using Mashup based e-Learning Content Collection and an Attractive Avatar in OpenSimulator
}

\author{
Kohei Arai \\ Department of Information Science \\ Saga University, Saga City, Japan
}

\begin{abstract}
Mashup based e-learning content collection and provide system with an attractive avatar in OpenSimulator is proposed for making learning processes attractive and finding the students week points through learning processes with avatar. Through experiments with under graduated students, it is found that the proposed e-learning system is useful for the under graduated students. The students may find their week points through learning processes. Avatar provides the questions of the student's week subjects. The students can enjoy the communications with their avatar. Therefore, the students have the most appropriate e-learning content which provided by the mashup based information retrievals and have lessons with their own avatar attractively using the proposed e-learning system.
\end{abstract}

Keywords-OpenSimulator; Mashup; e-learning; mobile learning; avatar; search engine

\section{INTRODUCTION}

An e-learning system is an information system for carrying out e-learning, and is roughly composed of "learning materials / learning materials" and a "learning management system" (LMS). Note that the term "e-learning" alone, which does not include a system, may mean an information system for elearning.

The users of the e-learning system are assumed to be "learners" and "teachers", and the functions for learners are different from the functions for teachers. Many e-learning systems have a "system administrator" (system administrator) of the e-learning system, and the system administrator may support learning activities and educational activities.

e-learning can be classified into two types, asynchronous type on-demand (self-learning type) and synchronous type ondemand (live type). For both types of e-learning system has some problems, (1) it is not easy to find an appropriate elearning content matching to learners' characteristics, (2) most of e-learning contents are not so attractive so that it is hard to continue their learning processes.

E-learning system utilizing learners' characteristics recognized through learning process with OpenSimulator ${ }^{1}$ is proposed [1]. Also, avatar utilized Q/A system of e-learning content designed with OpenSimulator is proposed and well reported [2].

\footnotetext{
${ }^{1}$ http://opensimulator.org/wiki/Main_Page
}

Module based content adaptation of composite e-learning content for delivering to mobile learners is proposed [3]. Eye based human-computer interaction allowing phoning, reading e-book/e-comic/e-learning, Internet browsing and TV information extraction is also proposed [4]. Efficiency improvements of e-learning document search engine for mobile browser is proposed and validated the system efficiency [5] together with e-learning document search method with supplemental keywords derived from keywords in meta-tag and descriptions which are included in the header of the first search result [6].

Method for leaning efficiency improvements based on gaze location notifications on e-learning content screen display is proposed [7] while video searching optimization with supplemental semantic keyword for e-learning video searching [8]. On the other hand, e-learning system which allows students' confidence level evaluation with their voice when they answer to the questions during achievement tests is well reported [9].

E-learning system utilizing learners' characteristics recognized through learning process with Open Simulator is proposed and evaluated its effectiveness [10]. Question answering for collaborative learning with answer quality predictor is proposed and validated [11]. Meanwhile, lecture's e-table (server terminal) which allows monitoring the location at which each student is looking during lessons with e-learning contents through client terminals is proposed and reported its effectiveness [12].

Development of learning support software with CG animations for intellectually disabled children is conducted and validated its effectiveness [13]. On the other hand, question answering for collaborative learning with answer quality prediction is proposed [14]. Free Open Source Software (FOSS) based e-learning system together with blended learning system is introduced as a cost effective system [15]. Predicting quality of answer in collaborative question answer learning is also proposed [16].

Yahoo! Search and web API utilized mashup based elearning content search engine for mobile learning is proposed and evaluated its effectiveness [17]. Meanwhile, eexperimental study of spatial recognition capability enhancement with building blocks learning contents for disable children is conducted and well reported [18]. 
In order for making learning processes attractive and finding the students week points through learning processes, an e-learning system using mashup based e-learning content retrievals and avatar (in OpenSimulator) for attractive lessons is proposed here. Learners can continue their lessons attractively using these OpenSimulator utilized e-learning system. On the other hand, mashup based e-learning content retrievals is getting much popular because it is easy to retrieve attractively and effectively and efficiently. Related contents are also provided from the mashup based content retrievals for overcoming week points of which avatar is found through lessons. Therefore, learners may choose the most appropriate e-learning contents together with the related contents easily.

The following section describes research background of OpenSimulator and mashup technologies followed by the proposed e-learning system. Then some examples of the proposed system as well as experimental results are described. After that, conclusion is described together with some discussions and with future research works.

\section{RESEARCH BACKGROUND}

The key components of the proposed e-learning system are OpenSimulator and Mashup content retrieval. Therefore, research background of the key components and software used are described in this section.

\section{A. Mashup}

API (Application Program Interface) is a set of instructions and functions that can be used when developing software.

For example, suppose you want to display characters in a window. If you have an API, you can use a function that displays characters in a window, but if you do not have an API, you can do it in dozens of lines (possibly hundreds, You may have to write a "function that displays characters in the window" yourself.

The mashup is to create a new service by combining technologies and contents from multiple different providers.

There are various technologies in the world, but each has its own strengths and weaknesses.

For example, yahoo's Yahoo map API displays a map based on latitude and longitude, and if you use the " Geocoding API" that can search for latitude and longitude from Google's address, site that displays a map of the input address " Can be made.

In this way, creating something by combining multiple technologies (APIs) is called mashup.

\section{B. OpenSimulator}

With the OpenSimulator, it is capable to create a virtual environment (virtual world), it is possible to access from various clients and multiple protocols, build own environment using technologies of the right materials, including the construction of avatars, etc. It can be an application server. In addition, it can be visually displayed by using a dedicated viewer. Open Sim is the main OpenSimulator.

OpenSimulator is a 3D application server. It is possible to create a virtual environment (virtual world) and access it from various clients and from multiple protocols. With OpenSimulator, it is capable to build own environment using the right technology at the right moment. - It is designed the software so that it is possible to customize any configuration of the building through the load module. OpenSimulator has been released under the BSD licens ${ }^{2}$ : Berkeley Software Distribution License, and it is open source but it is commercially available.

With no difficult settings, OpenSimulator can be used to simulate a virtual environment similar to Second Life $^{3}$ TM (client is also compatible). Other environments, protocols, and functions can be used by installing add - on modules. A list of available modules is on the Forge site ${ }^{4}$.

OpenSimulator is still alpha version software. Although OpenSimulator is relatively new software, it already has the following many useful functions:

1) It is capable to create multiple "worlds" in one application instance.

2) Multiple clients and protocols can be used - the same world can be accessed with multiple protocols at the same time.

3) It is possible to customize an avatar by custom made clothes, skins, wearing objects, and so on.

4) It can select from among several engines such as ODE (Open Dynamics Engine) ${ }^{5}$, and perform physical simulation in real time.

5) Using in - world construction tools, it can create content in real - time in the environment. "What you see is what you get" (what you see is what you get).

6) It is capable to create in-world applications in many languages including LSL / OSSL ${ }^{6}, \mathrm{C} \#, \mathrm{VB} \mathrm{NET}^{7}$, etc.

It is an OpenSimulator provided by OpenSimulator. Although OpenSim ${ }^{8}$ is still under development, it is becoming a 3D virtual world open source project that is drawing attention due to its high degree of completeness and its compatibility with Second Life ${ }^{9}$ Clients. A function to display and create 3D objects such as buildings, a function to simulate a change in time, a function to edit an avatar's appearance and to manage inventory, a function to control avatar's appearance between inventory and multiple avatars (Such as chat based on chat and instant messenger, voice chat with VOIP (Voice over Internet Protocol $)^{10}$, execution of animation and gesture, exchange of possessions), script support function, and so on.

The current release is 0.9.1.0 (released on October 28, 2019) and can be downloaded as

opensim-0.9.1.0.tar.gz or

opensim-0.9.1.0.zip

\footnotetext{
${ }^{2}$ https://opensource.org/licenses/BSD-3-Clause

${ }^{3}$ https://secondlife.com/?lang=ja-JP

${ }^{4} \mathrm{https}: / /$ secondlife.com/?lang=ja-JP

${ }^{5}$ http://demura.net/ode

${ }^{6}$ https://www.ossl.wiki/

${ }^{7}$ https://www.yumpu.com/en/document/view/43918605/opensimscripting-languages-lsl-and-ossl-justinccs-/3

${ }^{8}$ http://opensim.stanford.edu/

${ }^{9} \mathrm{https}: / /$ secondlife.com/?lang=ja-JP

${ }^{10}$ http://e-words.jp/w/VoIP.html
} 


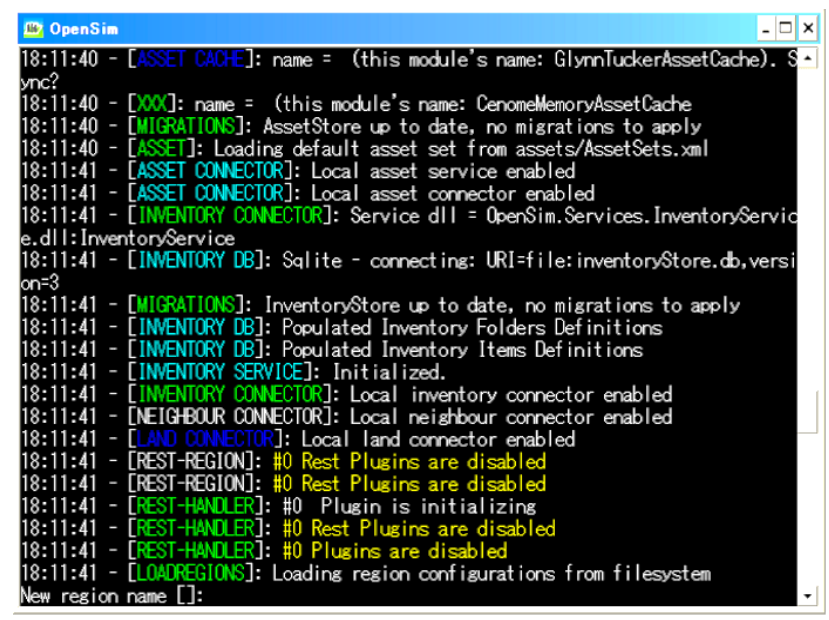

Fig. 1. OpenSimulator Description.

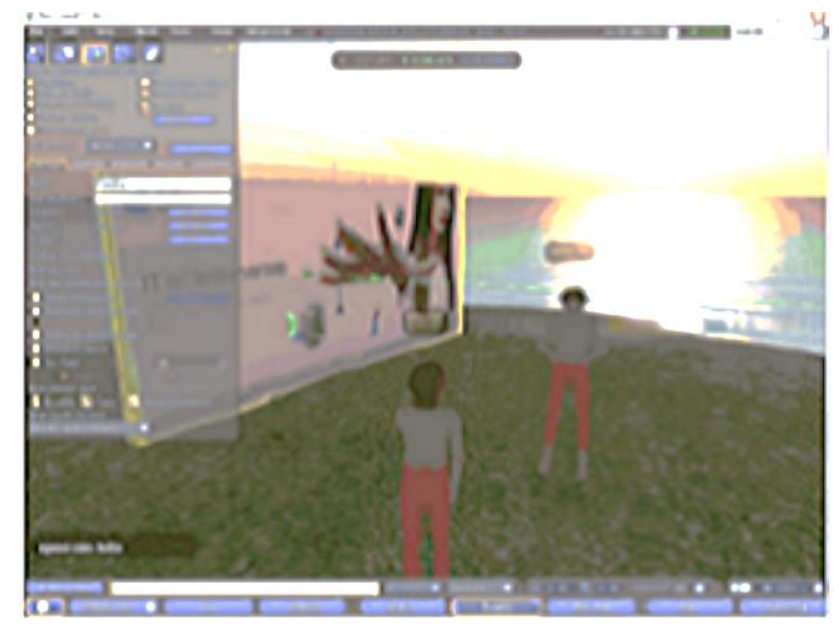

Fig. 2. Example Display is made by the Dedicated viewer of OpenSimulator.

Fig. 1 shows the OpenSimulator description. Meanwhile, an example of OpenSimulator environment is shown in Fig. 2. The example display is made by the dedicated viewer of OpenSimulator.

\section{Software used}

1) XAMPP: XAMPP is a package of free software necessary for running web applications, which is provided by apachefriends.org. Although it is mainly for development or learning, XAMPP is sometimes used as an actual operation environment on an intranet or the like. Apache (Web server), MySQL (SQL database server) and PHP which is the Web programming language and Perl ${ }^{11}$ used for same purpose, management tool such as phpMyAdmi ${ }^{12}$, and several auxiliary software such as SQLite Library modules are included. Originally, Linux was the only supported OS, it was called LAMPP $^{13}$ with its initial letter L, but since it corresponded to multiple OS later, changed L to $\mathrm{X}$ and became XAMPP.

\footnotetext{
${ }^{11} \mathrm{https}: / / \mathrm{www}$.activestate.com/activeperl/downloads

12 https://www.phpmyadmin.net/

${ }^{13} \mathrm{http}: / /$ seesaawiki.jp/w/tororo66/d/LAMPP
}

2) PHP: In this system, PHP was used for development of the Web interface, cooperation with the database, and creation of the program. PHP is a script language that is used by Hypertext Preprocessor, which is one of extended functions of a Web server that dynamically generates Web pages. It has features such as excellent XML support and cooperation with various databases, being an interpreter type scripting language, and being grammar similar to $\mathrm{C} / \mathrm{C}++$, Java, so it is easier to understand.

3) MySQL: Avatar e-learning MySQL was used as a database for storing data when learning content. MySQL is an open source relational database management system (RDBMS) developed by TCX DataKonsult $\mathrm{AB}^{14}$ and others. It is possible to perform retrieval processing and update processing at high speed and keeping the speed inferior to that of commercial DBMS. MySQL has a feature that it has high affinity with the Web, and it has a reputation as a service on the Internet and a back end system of the EC site. In addition, it is compatible with many platforms such as Windows and various UNIX type OS, and interfaces for various programming languages are prepared.

4) phpMyAdmin: phpMyAdmin is free software written in PHP that can manage MySQL via the Web. It is possible to perform various operations such as database, table, field, index, authority management, etc. on MySQL database system without describing SQL statement. Also, the user can write arbitrary SQL statement and execute it.

\section{PROPOSED E-LEARNING SySTEM}

\section{A. Design Concept}

In this system, the avatar himself accumulates a large amount of data, extracts appropriate data to the learner from the data, and presents it. For that purpose, we developed and studied with the following algorithm. First, learners do learning. Save the result data in the avatar. (Save to Database) This allows the avatar to understand what kind of person the learner is. Next, the avatar collects the information (data) of the e-learning site and saves it. This is the knowledge of avatar. Then, using the learning history of the learner, data suitable for the learner is extracted and presented. The result data is newly stored and accumulated. The basic process flow is shown in Fig. 3.

\section{B. Database Structure}

In this research, it is necessary to accumulate data in the database as accumulation of avatar knowledge. The structure of the database was constructed as shown in Fig. 4.

Meanwhile, the detailed database structure and the contents of the database are shown in Fig. 5(a) and (b), respectively. On the other hand, an example of the form screens is shown in Fig. 6. Designed as a form screen on the web browser as shown above. Create a button with the value you are not good at, and select it. Once, learners had a lesson, then their week subjects is analyzed by the proposed e-learning system through avatar gathers their past lesson leant and achievement test scores.

\footnotetext{
${ }^{14}$ https://www.easycounter.com/report/tcx.se
} 
Then, the week subjects are provided from the avatar as shown in Fig. 7.

The proposed e-learning system extract subjects which are not good from the correct answer rate of the learning history and present the problem of the subject to the learner. The learner inputs the answer in the text box and inputs the answer button. The response results are shown in Fig. 8. It says "Your problem is" " $1+1=$ ?" Then "Correct".

Compares the input value with the answer, presents the result to the learner, calculates the correct answer rate including past results on this question, and presents it to the learner. Save the result as the learning history and return to the form screen. Perform the same operation as before. When a certain percentage of correct answers are exceeded, a new problem is presented. (In this research, we set the correct answer rate at $80 \%$ or more.) Again, do the same operation as before.

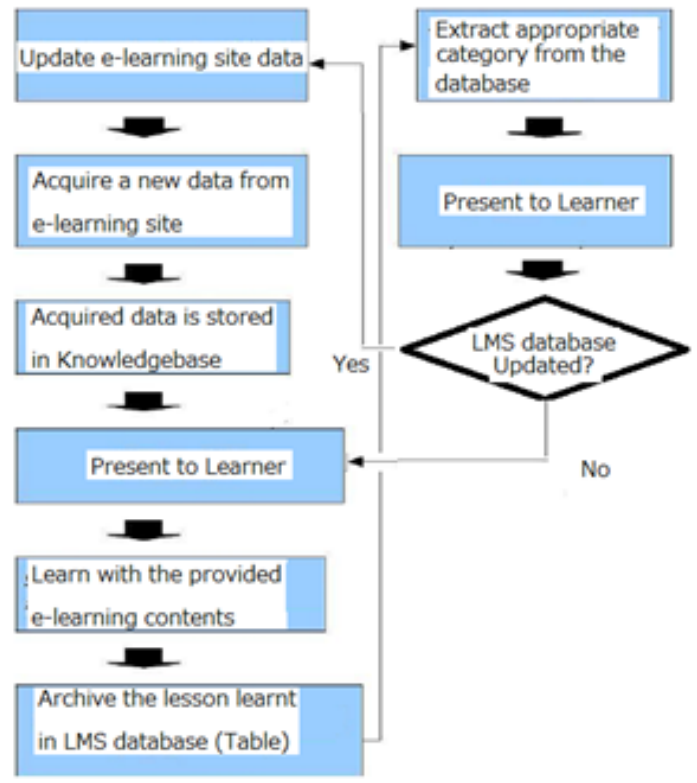

Fig. 3. Fundamental Process Flow of the Proposed e-Learning System.

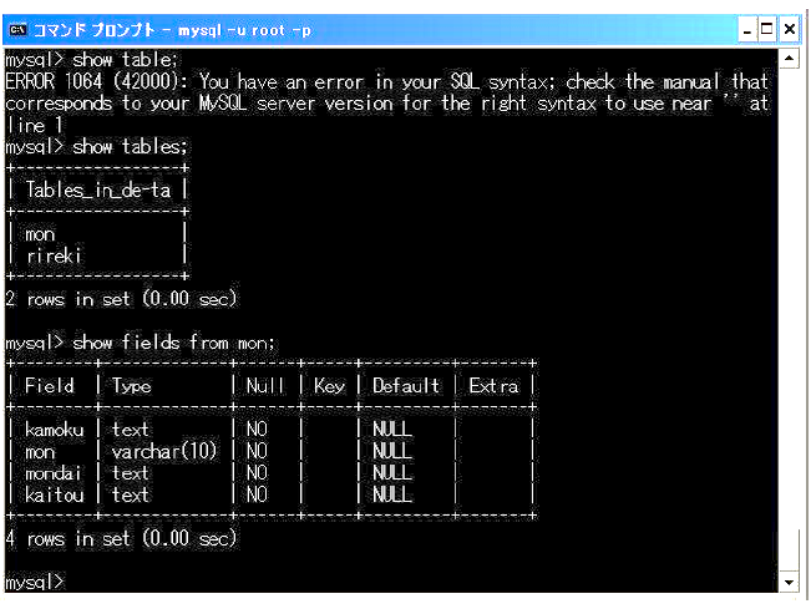

Fig. 4. Database Structure.

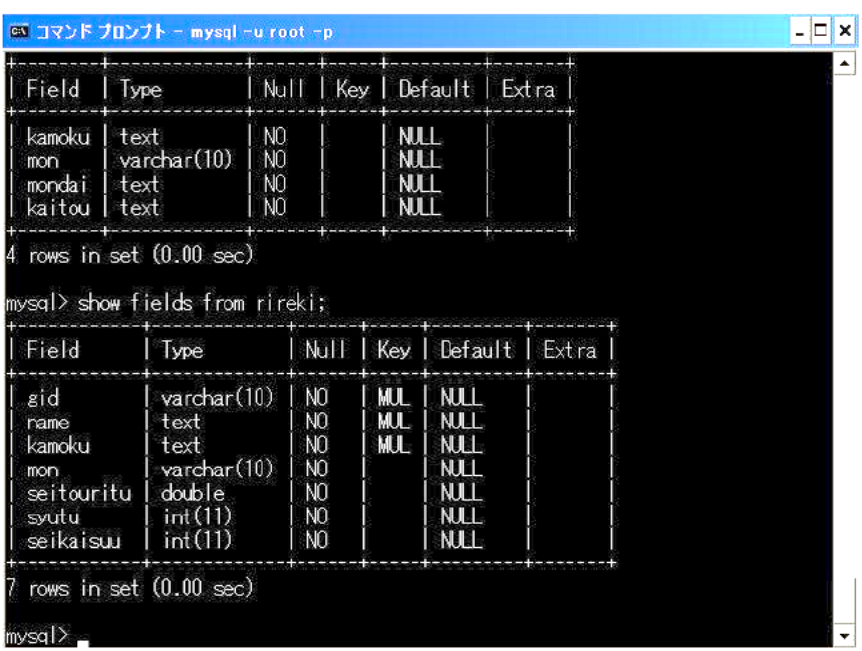

(a) Detailed Database Structure.

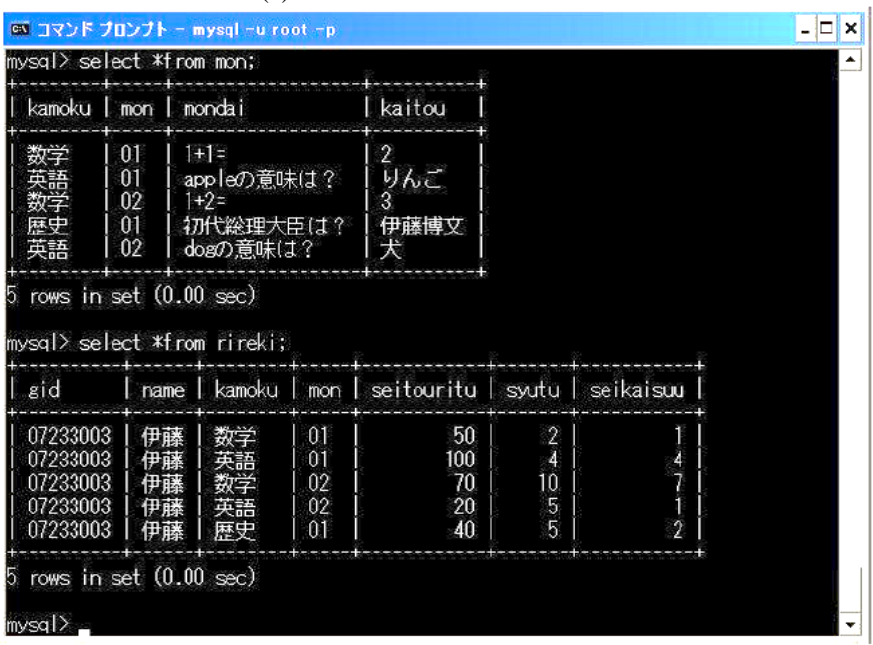

(b) Content.

Fig. 5. Contents in the Database.

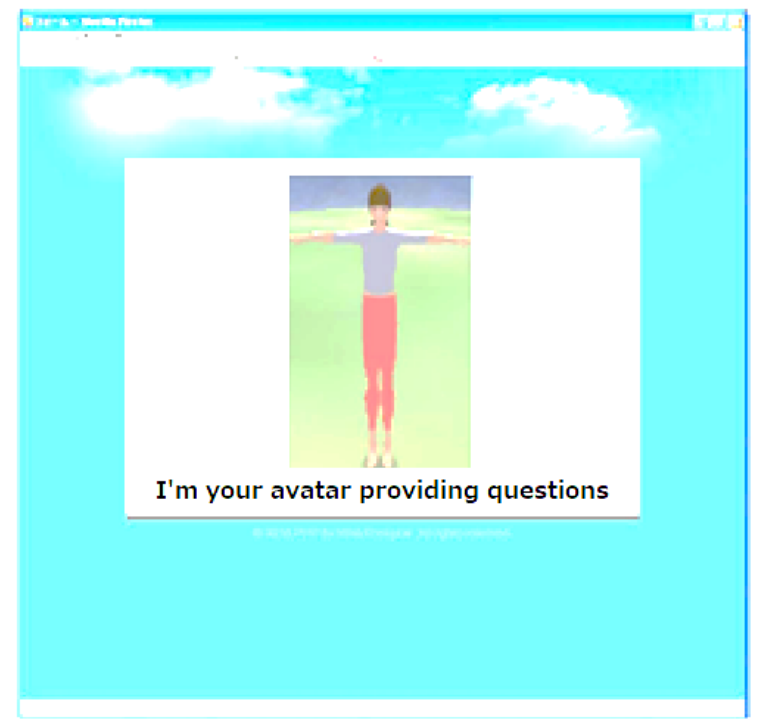

Fig. 6. Example of the form Screen. 


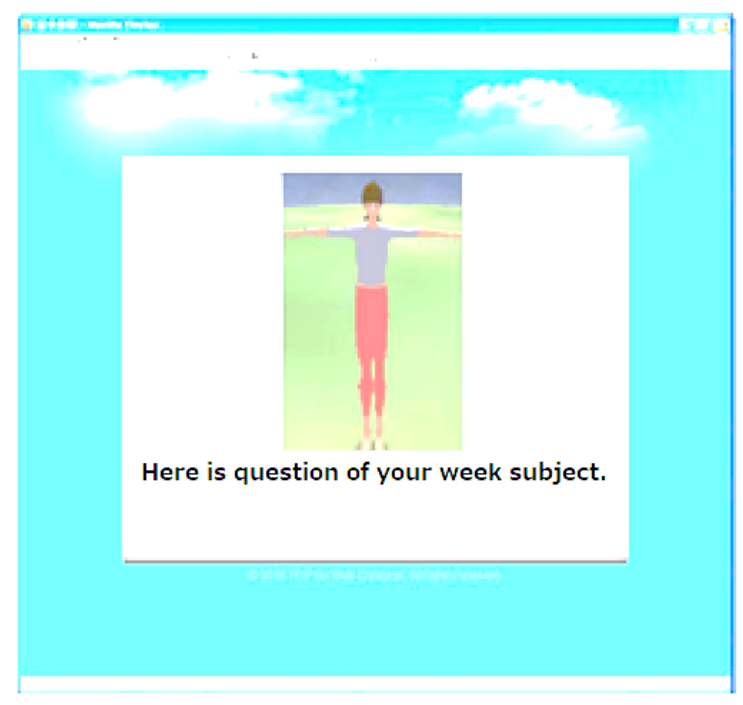

Fig. 7. Weak Subject Display.

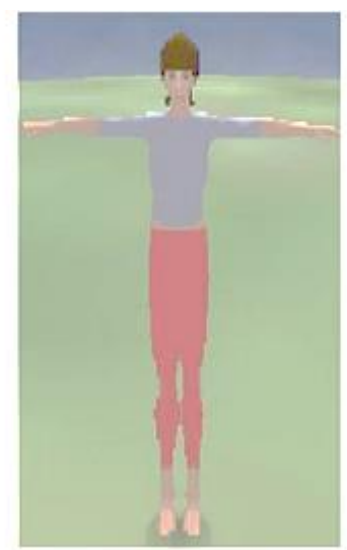

\section{Q.1+1=?}

2

\section{Correct!}

Fig. 8. Response Result.

If you answer incorrect values for the questions you have made, display as incorrect and display the correct answer. Furthermore, the correct answer rate including past results on this problem is calculated and presented to the learner as shown in Fig. 9. It says " The first Japanese prime minister?" "Incorrect Answer" "Correct answer is Hirofumi Itoh".

In this way, the proposed e-learning system save the learning history of the learner as data and present the problem suitable for the learner. Avatar accumulates learning history of learners, accumulates knowledge of avatars by acquiring data from e-learning site and accumulating data. And since avatar can extract and present mistakes learners caused in the past based on the data, learning can be done more efficiently than ever. In this time, although it was possible to extract data suitable for the learner, acquisition of data is incomplete.

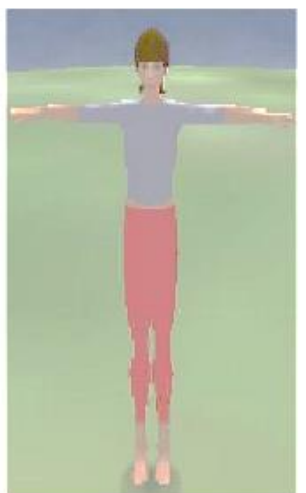

Who is the first prime ministerin Japan?

?

Correct answer is Hirofumi Ito

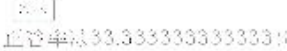

Fig. 9. Correct Answer Rate Including past Results on this Problem is Calculated and Presented to the Learner.

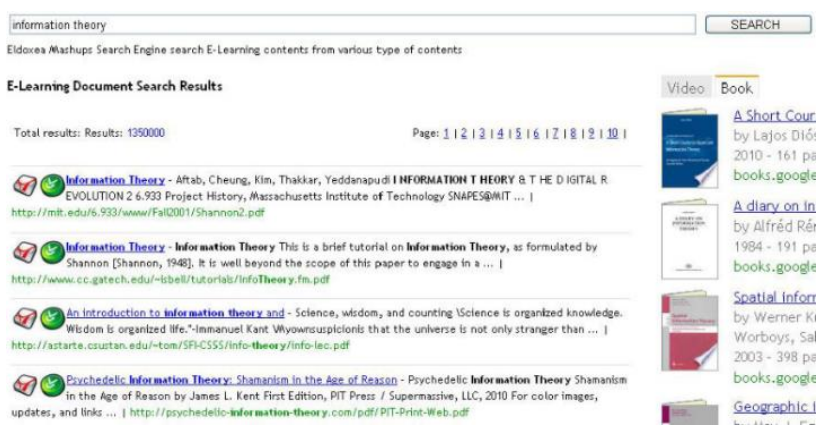

Fig. 10. Screenshot of an Example of e-Learning Content List Gathered with Mashup (Keyword = Information Theory).

\section{Mashup Contents}

A web mashup is a new type of web application that uses data and services from one or more external sources (usually from the Internet) to build entirely new and different web applications. Mashup web services is not a web portal. Combining Web service technologies with fresh content, collaborative approaches (such as Web $2.0^{15}$ technologies), and possibly Web data management and semantic technologies $\left(\mathrm{RSS}, \mathrm{RDF}^{16}\right.$ ).

Mashup is a new technology based on Web 2.0 and web service technology. Mashup create a new web application with combining data from two or more web services. Therefore, a variety of e-learning contents can be provided for in particular over coming week subject by using mashup technology. Fig. 10 shows screenshot of an example of e-learning content list gathered with mashup (keyword = Information Theory).

\section{EXPERIMENT}

The proposed e-learning system is used for 10 of the under graduated students in the information science department of Saga University. They learnt about mathematics (in particular, linear algebra), Physics (in particular Newtonian equation),

\footnotetext{
${ }^{15} \mathrm{https}: / /$ en.wikipedia.org/wiki/Web_2.0

${ }^{16}$ https://ja.wikipedia.org/wiki/RSS
} 
common social issues (in particular politics, economy) using the proposed e-learning system with their avatar for about 5 hours.

\section{Their impressions are as follows:}

1) My avatar tells my unknown week subject appropriately (the students may find their week points through learning processes),

2) Mashup e-learning contents are useful for overcoming week subjects (Avatar provides the questions of the student's week subjects)

3) The proposed e-learning system is much more attractive than the others because avatar is getting smarter through their learning processes (the students can enjoy the communications with their avatar).

\section{CONCLUSION}

Mashup based e-learning content collection and provide system with avatar in OpenSimulator is proposed. Through experiments with under graduated students, it is found that the proposed e-learning system is useful for the under graduated students. The students may find their week points through learning processes. Avatar provides the questions of the student's week subjects. The students can enjoy the communications with their avatar. Therefore, the students have the most appropriate e-learning content which provided by the mashup based information retrievals and have lessons with their own avatar attractively using the proposed e-learning system.

Further study is required for the followings,

1) Rethink the data acquisition algorithm again.

2) Implementation of data storage in the database.

3) Adapting this system to Open Simulator.

\section{ACKNOWLEDGMENT}

The author would like to tank Dr. Herman Tole of Brawijaya University, Indonesia and Mr. Kazuma Itoh of former student of the Saga University Japan for his effort to conduct the experiments.

\section{REFERENCES}

[1] Kohei Arai, Anik Nur Handayani, E-learning system utilizing learners' characteristics recognized through learning process with Open Simulator, International Journal of Advanced Research in Artificial Intelligence, 2, 4, 8-12, 2013.

[2] Kohei Arai, A.N.Hardiyani, Avatar utilized Q/A system of e-learning content designed with Open Simulator, Proceedings of the International Conference on Convergence Content 2012, 149-150, 2012.

[3] 254. Kohei Arai and Tolle Herman, Module based content adaptation of composite e-learning content for delivering to mobile learners, International Journal of Computer Theory and Engineering, 3, 3, 382387, 2011.

[4] Kohei Arai, Ronny Mardiyanto, Eye-based human-computer interaction allowing phoning, reading e-book/e-comic/e-learning, Internet browsing and TV information extraction, International Journal of Advanced Computer Science and Applications, 2, 12, 26-32, 2011.

[5] 295. Kohei Arai, Herman Tolle, Efficiency improvements of e-learning document search engine for mobile browser, International Journal of Research and Reviews on Computer Science, 2, 6, 1287-1291, 2011.
[6] Kohei Arai, T.Herman, E-learning document search method with supplemental keywords derived from keywords in meta-tag and descriptions which are included in the header of the first search result, International Journal of Advanced Computer Science and Applications, 3, 4, 99-104, 2012

[7] Kohei Arai, Method for leaning efficiency improvements based on gaze location notifications on e-learning content screen display, International Journal of Advanced Research in Artificial Intelligence, 1, 3, 1-6, 2012.

[8] 319. Kohei Arai, T.Herman, Video searching optimization with supplemental semantic keyword for e-learning video searching, International Journal of Research and Review on Computer Science, 3, 3, 1640-1644, 2012.

[9] Kohei Arai, E-learning system which allows students' confidence level evaluation with their voice when they answer to the questions during achievement tests, International Journal of Advanced Computer Science and Applications, 3, 9, 80-84, 2012.

[10] Kohei Arai, Anik Nur Handayani, E-learning system utilizing learners' characteristics recognized through learning process with Open Simulator, International Journal of Advanced Research in Artificial Intelligence, 2, 4, 8-12, 2013.

[11] Kohei Arai, Anik Nur Handayani, Question answering for collaborative learning with answer quality predictor, International Journal of Modern Education and Computer Science, 5, 5, 12-17, 2013.

[12] Kohei Arai, Lecture's e-Table (server terminal) which allows monitoring the location at which each student is looking during lessons with elearning contents through client terminals, International Journal of Advanced Research in Artificial Intelligence, 2, 6, 40-45, 2013.

[13] Kotaro Taguchi, Mariko Oda, Ou Kouno Seio Oda, Kohei Arai, Development of learning support software with CG animations for intellectually disabled children, Journal of Education System and Information Society of Japan, 30,1, 48-56, 2014.

[14] Kohei Arai, Anik Nur Handayani, Question Answering for collaborative learning with answer quality prediction, International Journal of Modern Education and Computer Science, 5, 5, 12-17, 2013.

[15] Kohei Arai, Free Open Source Software: FOSS based e-learning system together with blended learning system, International Journal of Advanced Research in Artificial Intelligence, 2, 11, 9-16, 2013.

[16] Kohei Arai, Anik Nur Handayani, Predicting quality of answer in collaborative question answer learning, International Journal of Advanced Research in Artificial Intelligence, 3, 2, 23-26, 2014.

[17] Kohei Arai, Yahoo! Search and web API utilized mashup based elearning content search engine for mobile learning, International Journal of Advanced Research on Artificial Intelligence, 4, 6, 1-7, 2015.

[18] Kohei Arai, Taiki Ishigaki, Mariko Oda, Experimental Study of Spatial recognition Capability Enhancement with Building Blocks Learning Contents for Disable Children, International Journal of Advanced Computer Science and Applications: IJACSA, 9, 6, 83-89, 2018.

\section{AUTHOR's PROFILE}

Kohei Arai, He received BS, MS and PhD degrees in 1972, 1974 and 1982, respectively. He was with The Institute for Industrial Science and Technology of the University of Tokyo from April 1974 to December 1978 also was with National Space Development Agency of Japan from January, 1979 to March, 1990. During from 1985 to 1987, he was with Canada Centre for Remote Sensing as a Post Doctoral Fellow of National Science and Engineering Research Council of Canada. He moved to Saga University as a Professor in Department of Information Science on April 1990. He was a councilor for the Aeronautics and Space related to the Technology Committee of the Ministry of Science and Technology during from 1998 to 2000. He was a councilor of Saga University for 2002 and 2003. He also was an executive councilor for the Remote Sensing Society of Japan for 2003 to 2005. He is an Adjunct Professor of University of Arizona, USA since 1998. He also is Vice Chairman of the Science Commission "A" of ICSU/COSPAR since 2008 then he is now award committee member of ICSU/COSPAR. He wrote 37 books and published 570 journal papers. He received 30 of awards including ICSU/COSPAR Vikram Sarabhai Medal in 2016, and Science award of Ministry of Mister of Education of Japan in 2015. He is now Editor-in-Chief of IJACSA and IJISA. http://teagis.ip.is.saga-u.ac.jp/index.html 